\title{
Molecular signatures of multiple myeloma progression through single cell RNA-Seq
}

\author{
Jin Sung Jang ${ }^{1}$, Ying Li ${ }^{2}$, Amit Kumar Mitra ${ }^{3}$, Lintao Bi ${ }^{4}$, Alexej Abyzov², Andre J. van Wijnen $\mathbb{E}^{5}$, Linda B. Baughn ${ }^{6}$, \\ Brian Van Ness, Vincent Rajkumar (10), Shaji Kumar $\mathbb{1}^{4}$ and Jin Jen ${ }^{1,6}$
}

\begin{abstract}
We used single cell RNA-Seq to examine molecular heterogeneity in multiple myeloma (MM) in 597 CD138 positive cells from bone marrow aspirates of 15 patients at different stages of disease progression. 790 genes were selected by coefficient of variation (CV) method and organized cells into four groups (L1-L4) using unsupervised clustering. Plasma cells from each patient clustered into at least two groups based on gene expression signature. The L1 group contained cells from all MGUS patients having the lowest expression of genes involved in the oxidative phosphorylation, Myc targets, and mTORC1 signaling pathways $\left(p<1.2 \times 10^{-14}\right)$. In contrast, the expression level of these pathway genes increased progressively and were the highest in $L 4$ group containing only cells from MM patients with $t(4 ; 14)$ translocations. A 44 genes signature of consistently overexpressed genes among the four groups was associated with poorer overall survival in MM patients (APEX trial, $p<0.0001 ; \mathrm{HR}, 1.83 ; 95 \% \mathrm{Cl}, 1.33-2.52$ ), particularly those treated with bortezomib ( $p<0.0001 ; \mathrm{HR}, 2.00 ; 95 \% \mathrm{Cl}, 1.39-2.89)$. Our study, using single cell RNA-Seq, identified the most significantly affected molecular pathways during MM progression and provided a novel signature predictive of patient prognosis and treatment stratification.
\end{abstract}

\section{Introduction}

Multiple myeloma (MM) is a malignant hematological disorder characterized by the accumulation of terminally differentiated antibody-secreting plasma cells with clonal genetic/cytogenetic abnormalities that home to the bone marrow $^{1-3}$. Clinically, monoclonal gammopathy of undetermined significance (MGUS) is a pre-neoplasic condition preceding MM. Extensive immunophenotypic and differential gene expression analyses have shown that MGUS and MM can also be distinguished from normal plasma cells but not from each other ${ }^{4}$.

Fluorescence in situ hybridization (FISH) studies of neoplastic plasma cells demonstrate trisomies of multiple

\footnotetext{
Correspondence: Shaji Kumar (Kumar.Shaji@mayo.edu) or Jin Jen (jen.jin@mayo.edu)

${ }^{1}$ Genome Analysis Core, Medical Genome Facility, Center for Individualized Medicine, Mayo Clinic, Rochester, MN, USA

${ }^{2}$ Division of Bioinformatics and Biostatistics, Department of Health Science Research, Mayo Clinic, Rochester, MN, USA

Full list of author information is available at the end of the article.

These authors contributed equally: Jin Sung Jang, Ying Li
}

odd numbered chromosomes in $40 \%$ of MM cases, while the majority of the remaining cases have a translocation involving the immunoglobulin heavy chain $(\mathrm{IgH})$ gene at chromosome $14 \mathrm{q} 32^{1-3}$. Secondary cytogenetic abnormalities can also occur during the disease progression, including gains of 1q, deletions of $17 \mathrm{p}$ (resulting in TP53 loss) and 13q, as well as mutations and secondary translocations involving $M Y C^{1,2}$. Both primary and secondary cytogenetic abnormalities, as well as specific molecular alterations are known to influence disease progression, response to therapy, and prognosis ${ }^{1}$. There is strong evidence of genetic heterogeneity based on the diverse pattern of molecular changes including clonal evolution and differential clonal response, which impact prognostic stratification, therapeutic approaches, and disease response to treatment ${ }^{2,5,6}$. High throughput single cell RNA-Seq (scRNA-Seq) technology offers an opportunity for an unbiased gene expression profiling of plasma cells obtained from each patient to understand the pathogenesis of MM progression that can better guide patient 
Table 1 Patient characteristics and distribution of cells based on 790 genes

\begin{tabular}{|c|c|c|c|c|c|c|c|}
\hline Sample IDs & $\begin{array}{l}\text { Total number of } \\
\text { cells sequenced }\end{array}$ & $\begin{array}{l}\text { Total number of cells } \\
\text { passing } Q C \text { and } \\
\text { analyzed }\end{array}$ & L 1 & L 2 & L3 & L4 & $\begin{array}{l}\text { Cytogenetic abnormality } \\
\text { information }\end{array}$ \\
\hline IgM MGUS1 & 26 & $24(92 \%)$ & $15(63 \%)$ & $6(25 \%)$ & $3(13 \%)$ & & Not tested \\
\hline IgM MGUS3 & 33 & $17(52 \%)$ & $16(94 \%)$ & $1(6 \%)$ & & & Not tested \\
\hline MGUS5 & 19 & $7(37 \%)$ & $4(57 \%)$ & $3(43 \%)$ & & & Normal \\
\hline SMMO & 84 & 77 (92\%) & & $10(13 \%)$ & & 67 (87\%) & $t(4 ; 14)$, gain $1 q 21$, del $13 q$ \\
\hline SMM2 & 40 & $16(40 \%)$ & $14(88 \%)$ & $2(12 \%)$ & & & $t(14 ; 20)$, monosomy 13 \\
\hline SMM3 & 40 & $39(98 \%)$ & & $4(10 \%)$ & $34(90 \%)$ & & Trisomy 7, 9, 11 and 15 \\
\hline SMM4 & 51 & $44(86 \%)$ & & $18(41 \%)$ & $26(59 \%)$ & & Trisomy $3,7,9,11,14$, \& 15 \\
\hline NDMM3 & 65 & $59(91 \%)$ & $3(5 \%)$ & $27(46 \%)$ & $29(49 \%)$ & & $\begin{array}{l}\text { Trisomy } 3,7, \& 11 \text {, trisomy/ } \\
\text { tetrasomy } 9 \& 15\end{array}$ \\
\hline NDMM5 & 41 & $32(78 \%)$ & $1(3 \%)$ & $30(94 \%)$ & $1(3 \%)$ & & $\begin{array}{l}\text { Trisomy } 7,9,11, \& 14 \text {, trisomy/ } \\
\text { tetrasomy } 3 \& 15 \text {, del } 13 q\end{array}$ \\
\hline NDMM6 & 48 & $47(98 \%)$ & $3(6 \%)$ & $41(88 \%)$ & $3(6 \%)$ & & Trisomy $3,9,11, \& 15$ \\
\hline NDMM7 & 59 & $54(92 \%)$ & $30(56 \%)$ & $24(44 \%)$ & & & $t(11 ; 14)$ \\
\hline NDMM8 & 63 & $60(95 \%)$ & & $26(43 \%)$ & $34(57 \%)$ & & $\begin{array}{l}\text { Trisomy } 3,8,9, \& 14 \text {, trisomy/ } \\
\text { tetrasomy } 7 \text {, tetrasomy } 11 \text {, gain } \\
\text { 1q21 }\end{array}$ \\
\hline RRMM1 & 48 & $46(96 \%)$ & & $1(2 \%)$ & & 45 (98\%) & $t(4 ; 14)$, monosomy 13 , del $17 p$ \\
\hline RRMM2 & 50 & $42(84 \%)$ & $1(2 \%)$ & $12(28 \%)$ & $28(67 \%)$ & $1(2 \%)$ & $\begin{array}{l}t(4 ; 14) \text {, trisomy } 11 \& 15, \text { monosomy } \\
9 \& 13\end{array}$ \\
\hline RRMM4 & 34 & $33(97 \%)$ & $1(3 \%)$ & $32(97 \%)$ & & & $t(11 ; 14)$ and tetraploid \\
\hline Total & 701 & $597(85 \%)$ & $89(15 \%)$ & $237(40 \%)$ & $158(26 \%)$ & $113(19 \%)$ & \\
\hline
\end{tabular}

prognosis and selection for appropriate clinical interventions ${ }^{7}$.

In this study, we performed scRNA-Seq using 597 cells derived from 15 patients at different stages of $\mathrm{MM}$ including MGUS, smoldering multiple myeloma (SMM), newly diagnosed MM (NDMM), and relapsed and/or refractory MM (RRMM). At the resolution of single cells, we identified gene expression signatures and molecular pathways relating to disease progression that are present within each patient and affect overall survival (OS).

\section{Materials and methods}

MM samples, plasma cell selection, and cell capture for sCRNA-Seq

Bone marrow aspirates were collected from 15 patients (Table 1) after informed consent and subjected to ACK lysis and mononuclear cell isolation. Plasma cells were separated by positive selection using CD138-coated magnetic beads (MACS; Miltenyi Biotec, CA) in a RoboSep system (STEMCELL Technology, Canada). CD138-positive cells were examined using Vi-CELL XR (Beckman Coulter, CA) to determine cell number, viability, and average size. A microfluidic mRNA-Seq chip (Fluidigm, CA) was used for capturing cells from each sample at a concentration of 500 cells/ $\mu$ l and run in the Fluidigm $\mathrm{C} 1$ system to generate double-stranded cDNA using SMARTer Ultra Low RNA kit for Illumina (Takara, CA). All samples were assessed for cell capture in a C1 chip by direct observation under a microscope and for cDNA quality using Fragment Analyzer (HS Large Fragment kit, Advanced Analytical Technologies, IA). In total, 701 single cells that generated cDNA fragments $>1000 \mathrm{bp}$ on average were included in the subsequent sequencing analysis. While the exact protocol was used, we observed a significant variation on the total number of cells isolated from each bone marrow biopsy and the number of cells captured by the Fluidigm C1 chip. This is in part due to individual sample variations, as well as possible difference in disease stage, since the total number of captured plasma cells was overall lower in MGUS cases compared to those at SMM or MM stages (Table 1). Overall, the number of analyzable cells from each patient reflected the total number of CD138-positive cells available, as well as the quality of the bone marrow biopsy. Figure 1a outlines 


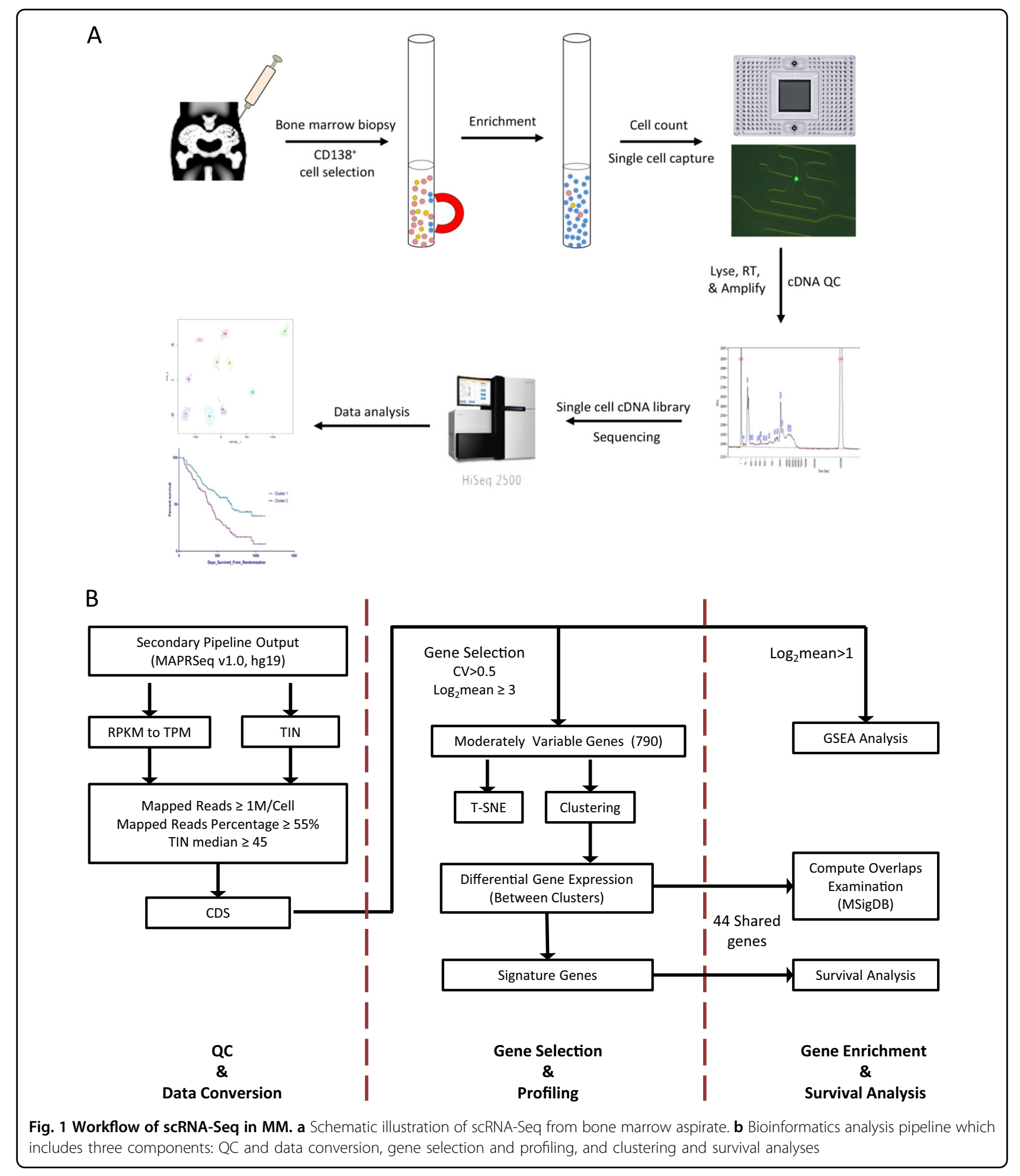

the major steps of this study. Aliquots of the same bone biopsy were also retained and analyzed by FISH as a part of the routine clinical diagnosis and extracted from patients' pathology record following institutional approved IRBs.

\section{scRNA-Seq library construction and sequencing}

For RNA-Seq library construction, single cell cDNAs $(250 \mathrm{pg})$ were used to construct indexed libraries using Nextera XT DNA Sample Preparation kit (Illumina, CA). Libraries were quantified by Bioanalyzer (High Sensitivity 
DNA analysis kit, Agilent, CA) and Qubit (dsDNA BR Assay kits, Life Technologies, CA). Single cell libraries obtained from each patient were pooled at up to 48 cells per lane and sequenced using the 101 bases paired-end protocol on Illumina HiSeq 2500 Rapid Run. FASTQ formatted raw files from each sample were mapped to the hg19.

\section{scRNA-Seq data QC and analysis}

We used MAPRSeq (v1.0) $)^{8}$ to analyze RNA-Seq data as outlined in Fig. 1b with TopHat2 for reads alignment to the hg19 and FeatureCounts for gene expression. During quality control (QC), we consider cells of low quality if the total number of reads/cell $<1,000,000$, percentage of mapped reads $<55 \%$, and if the median of non-zero gene transcript integrity number (TIN) $<45^{9}$. Of the 701 single cell libraries sequenced, 597 passed QC and were included in the downstream analysis. We observed that some genes have reads mostly aligned to the 3'UTR with few reads aligned to the coding DNA sequence (CDS) regions. To reduce bias in gene expression based on $3^{\prime} \mathrm{UTR}$ alignments, we used only expression in CDS for subsequent analysis ${ }^{10}$. Transcripts per kilobase million (TPM) was used as the measure for gene expression. Raw sequence data and processed data sets from this study have been submitted to Gene Expression Omnibus (GEO; http:// www.ncbi.nlm.nih.gov/geo/) under accession number GSE118900).

For gene selection and molecular signature analyses, we first performed Seurat $t$-distribution stochastic neighborhood embedding ( $t$-SNE, V1.2) and clustering analysis $^{11}$ using genes expressed in more than two cells with $\log _{2}$ mean $>1$ and standard deviation $(y)>1$ (Supplemental Figure S1). Secondly, we employed a coefficient of variation $(\mathrm{CV})^{12}$ approach to select for highly variable genes with $C V \geq 0.5$ and $\log _{2}(\mathrm{TPM}+1) \geq 3$ as the cutoff. Of the 790 genes in this group (Supplemental Table S1), $14.8 \%(n=117)$ were housekeeping genes (HGs) as categorized by Hsiao et al. ${ }^{13}$. In contrast, $40 \%(n=39)$ were HGs among the 99 genes with the high expression levels $\left(\log _{2}(\mathrm{TPM}+1)>3\right)$ and low $\mathrm{CV}$ values $\left.\mathrm{CV} \leq 0.5\right)$. Using this approach, we excluded HGs uniformly expressed in a majority of the cells while reducing the stochastic noise associated with low copy transcripts to capture the most significant differential gene expression signatures among the individual MM cells (Fig. 2a).

Unsupervised hierarchical clustering was performed based on the expression levels of the 790 selected genes using the "1-Pearson correlation" distance and Ward.D2 linkage approach. ANOVA analysis was performed among the major groups of cells $\left(\log _{2}\right.$ scale) and the significantly differentially expressed genes were selected using ANOVA $p$-value $<0.05$ and two-fold change (FC) (Supplement Figure S2). To further understand the biological characteristics of the data set, Compute Overlaps Examination of Molecular Signatures Database v5.2 (MsigDB) was carried out using 311 common genes and 44 signature genes that are significantly up-regulated $(\mathrm{FC} \geq 2, p<0.05)$ within each comparison using L1 as reference. Hallmarks gene set and C5 GO gene sets in MsigDB (v5.2) were used to identify the most significant pathways within the data set.

\section{Survival analysis using publically available datasets}

We extracted microarray gene expression (GEP) data from APEX trial ${ }^{14,15}$ on 44 with consistently increased expression among L1-L4 groups. For genes with multiple probe sets, mean intensities were computed. GEP data was then mean-centered and scaled prior to analysis. Unsupervised $K$-means clustering was performed using Hartigan and Wong's algorithm ${ }^{16}$ based on the GEP signature. Kaplan-Meier (KM) survival curves were generated for the clusters by computing OS over time. The KM curves were compared and $p$-values were generated using Mantel-Cox log-rank test.

\section{Results \\ Patient population and clinical status}

Our scRNA-Seq analysis included CD138-positive cells isolated from bone aspirates of patients with MGUS ( $n=$ $3)$, SMM $(n=4), \operatorname{NDMM}=5$, and RRMM $(n=3)$. A total of 701 cells were collected using the Fluidigm C1 nanofluidics platform and 597 cells passed QC and included in the analyses. An average of 16 cells from three MGUS patients were included in the analysis (range, 7-24 cells/ patient) while an average of 46 cells/patient were analyzed for those at either SMM or MM stages of the disease (range, 16-77 cells/patient and 40-50 cells/MM subgroup). Consistent with the clinical status of the disease, MGUS cases collectively had fewer analyzable CD138positive plasma cells compared those in later stages. A summary of the clinical information and FISH results obtained as a part of routine clinical practice for all patients are shown in Table 1.

\section{Gene selection and molecular classification of MM based on scRNA-Seq}

We first used $t$-SNE analysis to assess cell-to-cell variability after sample QC of scRNAseq data and observed that most plasma cells clustered primarily by individual patients reflecting the highly clonal nature of MM, except MGUS group (Supplemental Figure S1). We next used CV method to select 790 genes with moderately high expression values of $\log _{2}(\mathrm{TPM}+1) \geq 3$ and $\geq 0.5$ fold variation (CV) in gene expression across all 597 cells from 15 patients (Fig. 2a). By unsupervised hierarchical clustering (Fig. 2b), cells self-organized into four main clusters (L1-L4) each composed of cells from patients at different 


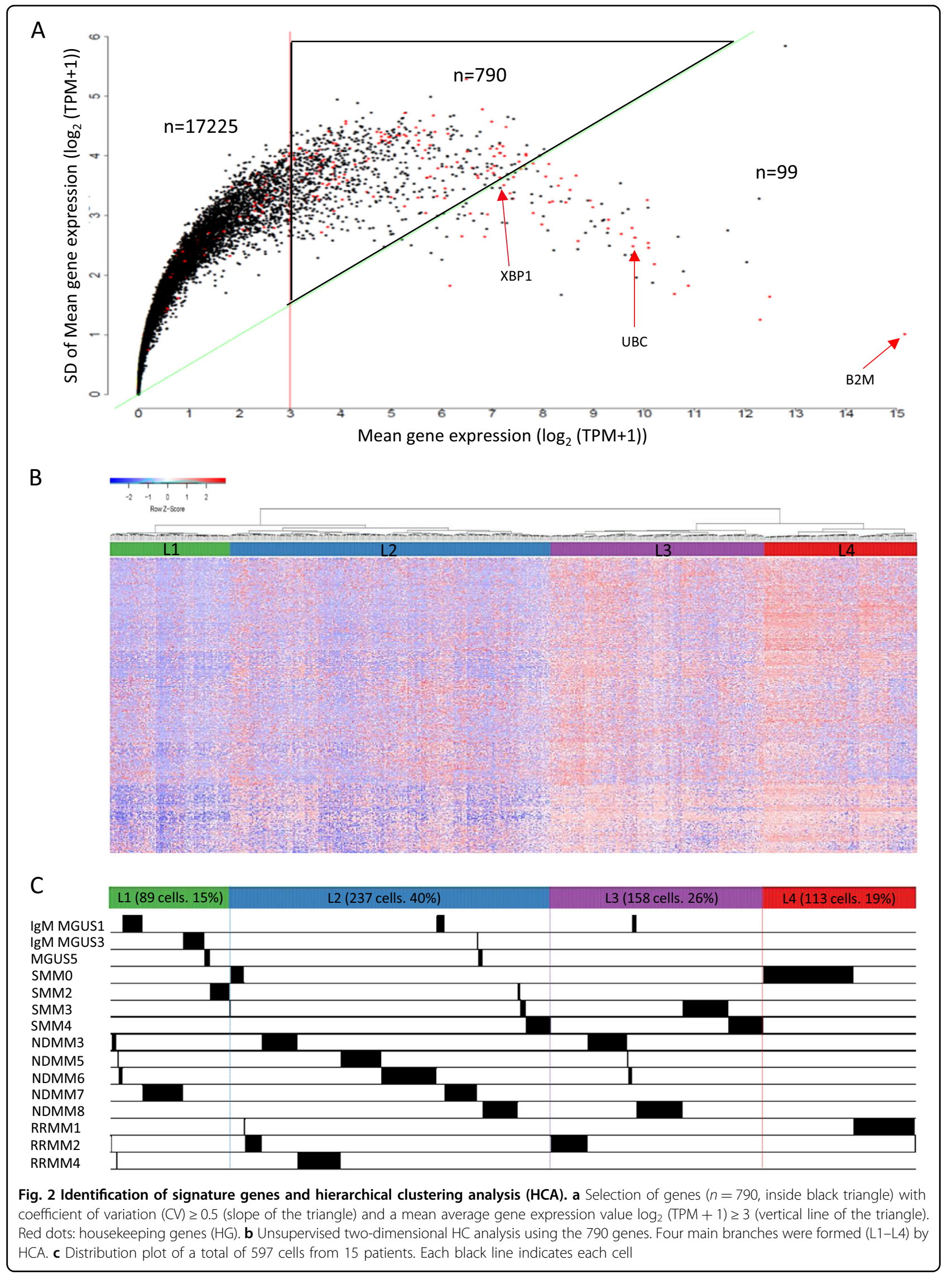


stages of MM diagnosis (Fig. 2b). Cells in the L1 group were characterized by low level expression in genes involved in the oxidative phosphorylation, Myc targets, and mTORC1 signaling when compared to the other groups $\left(p<1.2 \times 10^{-14}\right.$, Supplemental Table S2).

As shown in Table 1, most CD138-positive cells from the three MGUS patients clustered into the L1 group (63\%) with other cells in the L2 or L3 groups (29\% and 9\%, respectively). When compared with cytogenetic abnormalities identified by FISH, all cells from patients with either $t(14 ; 20)$ or $t(11 ; 14)$ translocation clustered into L1 and L2 groups (SMM2, NDMM7, and RRMM4). In contrast, a majority of cells from patients with trisomy features (SMM3 and 4, NDMM3, 5, 7, and 8) belonged to the L2 and L3 groups while cells with $t(4 ; 14)$ translocations appeared to contribute exclusively to the L4 cluster (SMM0, RRMM1 and 2). Sample RRMM2, with both $t(4 ; 14)$ and trisomies of 11 and 15 , had cells that cluster across all four clusters. In total, 89 cells formed L1 cluster (15\%) with a gene expression signature representing lowrisk MM having a high representation of cells from MGUS and cytogenetically favorable patients. Gene expression profiles from 237 cells in the L2 group (40\%) and 113 cells in L3 (26\%) more closely represented cytogenetically complex MM based on their association with cases having trisomies or tetrasomies. Lastly, gene expression signature from cells in the L4 group (113 cells, $19 \%)$ appeared to reflect the highest risk MM given it is exclusively of cells obtained from the patients having the $t(4 ; 14)$ translocation (Fig. 2c and Table 1).

\section{Altered protein homeostasis genes among subgroups}

Since it is well established that MM patients often respond well to proteasome inhibitor therapy, we examined the expression status of all 18 genes encoding the proteasome subunits in the 790 selected genes. Each of these genes was differentially over expressed when compared between the groups (Fig. 3a). At $p<0.05$, seven proteasome genes (PSMA2, PSMA4, PSMB1, PSMB3, PSMB7, PSMD7, and PSME2) were significantly highly expressed in L2 (FC, 2.2-13.7) compared to L1, while all 18 genes were significantly up-regulated in both L3 and L4 groups (FC, 3.0-40.4) when compared to cells in L1 group (Supplemental Table S3). Similarly, when comparing the L3 and L4 groups to L2, a majority of the proteasome genes were highly expressed in the later groups (FC, 2.6-22.3). Furthermore, eight proteasome genes (PSMA6, PSMB1, PSMB3, PSMB6, PSMB9, PSMC4, $P S M D 7$, and $P S M E 2)$ were expressed at remarkably higher levels (FC, 3.0-7.2) in L4 compared to L3. Additionally, critical genes involved in the unfolded protein response pathway (UPR, also known as ER stress response) were also significantly up-regulated. One of those, $X B P 1$ is stably expressed at high levels in a majority of the cells analyzed ( $\mathrm{CV} \leq 0.5$ and Fig. 2a). ATF6, a transcription factor that activates target genes for UPR, was predominantly expressed at a high level in the L4 group while EIF2A (Eukaryotic translation Initiation Factor $2 \mathrm{~A}$ ) was significantly highly expressed in both the L3 and L4 groups (Fig. 3b).

\section{Molecular pathways involved in MM progression}

Comparing cells in the L1 group to each of the higher cell clustering groups (L2-L4), we obtained a total of 311 common genes most significantly up-regulated from L1 to L4 groups $(p<0.05, \mathrm{FC} \geq 2$, Fig. 4a and Supplemental Table S4). Compute Overlaps Examination of MSigDB showed that gene sets shared among these groups were associated with cell metabolism and protein homeostasis, such as oxidative phosphorylation, Myc-targeted genes, mTORC1 signaling, and UPR (Fig. 4a). When considering genes significantly altered in expression levels $(\mathrm{FC} \geq 2, p<$ 0.05 ) between the adjacent groups, out of 311 common genes, we identified a 44 signature genes with consistently increased expression level among the groups (Fig. 4b). Using GO term analysis, we found that 26/44 (59\%) were related genes with UPR pathway, function of endoplasmic reticulum and mitochondria that highlighting their role in MM (Supplemental Table S5).

\section{Clinical implications of genes associated with MM progression}

We examine the clinical association of the 44 genes most consistently associated with MM progression from pair-wise comparisons between the four groups (L1 vs. L2, L2 vs. L3, and L3 vs. L4) to examine whether the expression patterns of these genes correlate with OS in MM patients. Using the APEX trial data set and when dichotomized as high and low expression groups, the 44 gene expression signature was able to distinguish $O S$ in all patients $(p<0.0001$; hazard ratio (HR), 1.831; 95\% CI, 1.33-2.522). Strikingly, this survival significance was primarily observed in the bortezomib treatment group ( $p<$ 0.0001 ; HR, 2.001; 95\% CI, 1.387-2.888) but not in patients treated with dexamethasone $(p<0.0812$; HR, 1.763, 95\% CI, 0.9133-3.403; Fig. 5).

\section{Discussion}

Single cell RNA-Seq is a powerful tool to identify unique cell types and unmask the cellular heterogeneity in the tumor microenvironment ${ }^{17,18}$. However, scRNA-Seq data can be inherently noisy due to pre-amplification of single cell RNA and the stochastic nature of RNA transcription ${ }^{19,20}$. Data analysis to identify underlying biological variations with confidence is further confounded by the large gene expression variations within a cell, and the lower coverage per transcriptome in general when the total reads are distributed over a large number of 


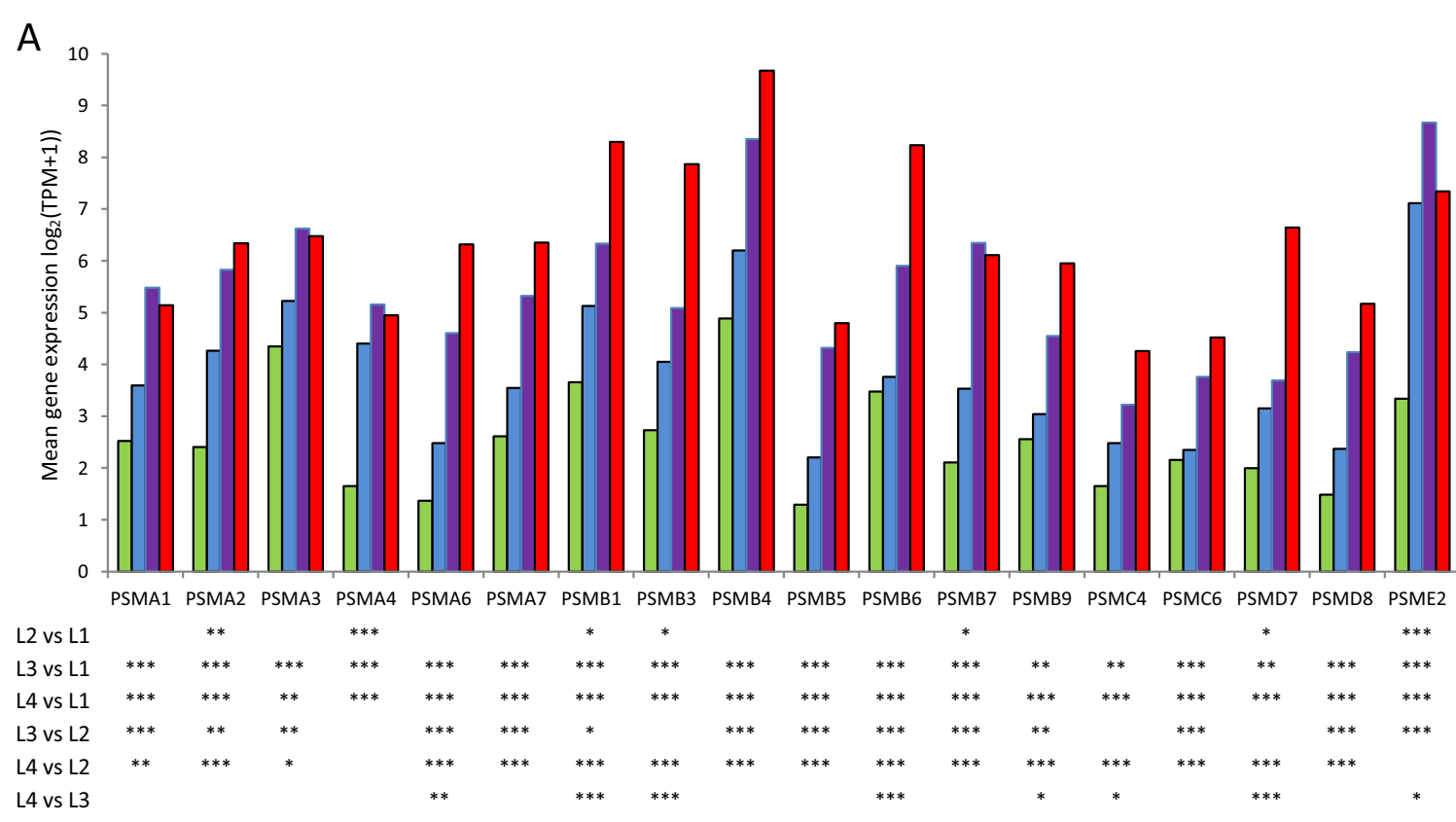

B

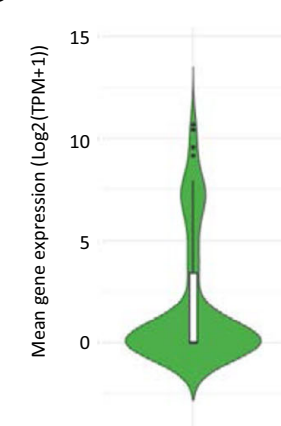

L1
ATF6

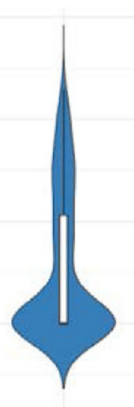

L2

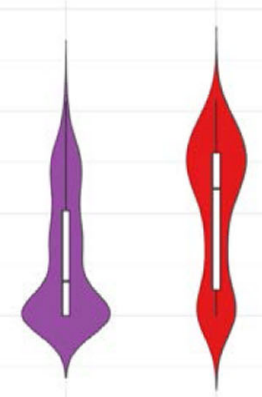

L3

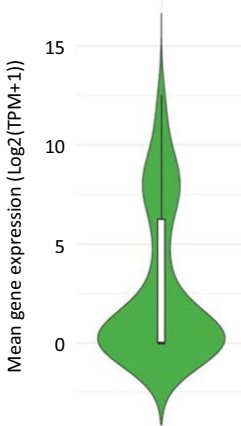

L1

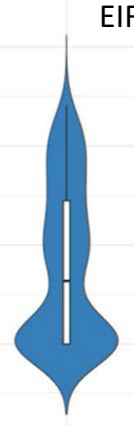

L2
EIF2A

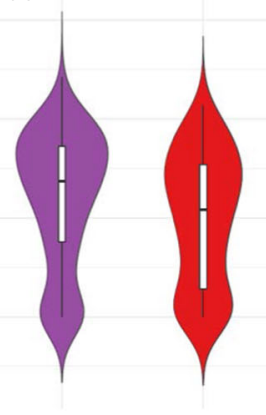

L4

Fig. 3 Expression of protein homeostasis genes among clustering cell groups. a Relative expression for 18 proteasome subunits genes in $L 1-L 4$ groups. $p$-values and fold changes by ANOVA are shown in Supplemental Table S3. b Relative expression of ATF6 and EIF2A genes within each single cell group. Vertical axis is the log-transformed mean expression values and width indicates frequency of cells at the indicated expression level. ${ }^{*} p<$ $0.05 ;{ }^{* *} p<0.01 ;{ }^{* * *} p<0.001$

individual cells rather than a single mixed cell population. In the context of MM, most transcriptome profiling studies to date have focused on CD138-selected plasma cells from bone marrow aspirates. Gene expression changes from pooled cells represent an average expression and could mask gene expression signatures by subpopulations of cells with high expression ${ }^{18,21-23}$. In addition, the highly monoclonal nature of the MM disease posts a significant challenge in assessing intercellular heterogeneity even at the resolution of single cells.

To overcome these technical challenges, we utilized several different analytical approaches for gene expression analysis in single cells. By $t$-SNE ${ }^{11}$ we observed that most cells clustered exclusively by individual patients reflecting the clonal genetic changes unique to each patient. We used the CV approach ${ }^{12}$ to focus on robustly expressed genes with a variation of $\mathrm{CV} \geq 0.5(n=790)$, thus reducing technical and biological noises for subsequent unsupervised clustering analyses. Using this strategy, we observed that cells from all 15 patients re-clustered into four sub-populations (L1-L4) based on the gene expression obtained by scRNA-Seq (Table 1). Cells derived from each patient's bone biopsy dispersed across different clusters reflecting the heterogeneous nature of plasma cells in MM (Fig. 2b).

A novel observation from our study is that while a highly clonal disease, plasma cells derived from each of the 15 patients have diverse molecular signatures that corresponded to different stages of disease and reflected cytogenetic features observed in $M M$ at different risk 

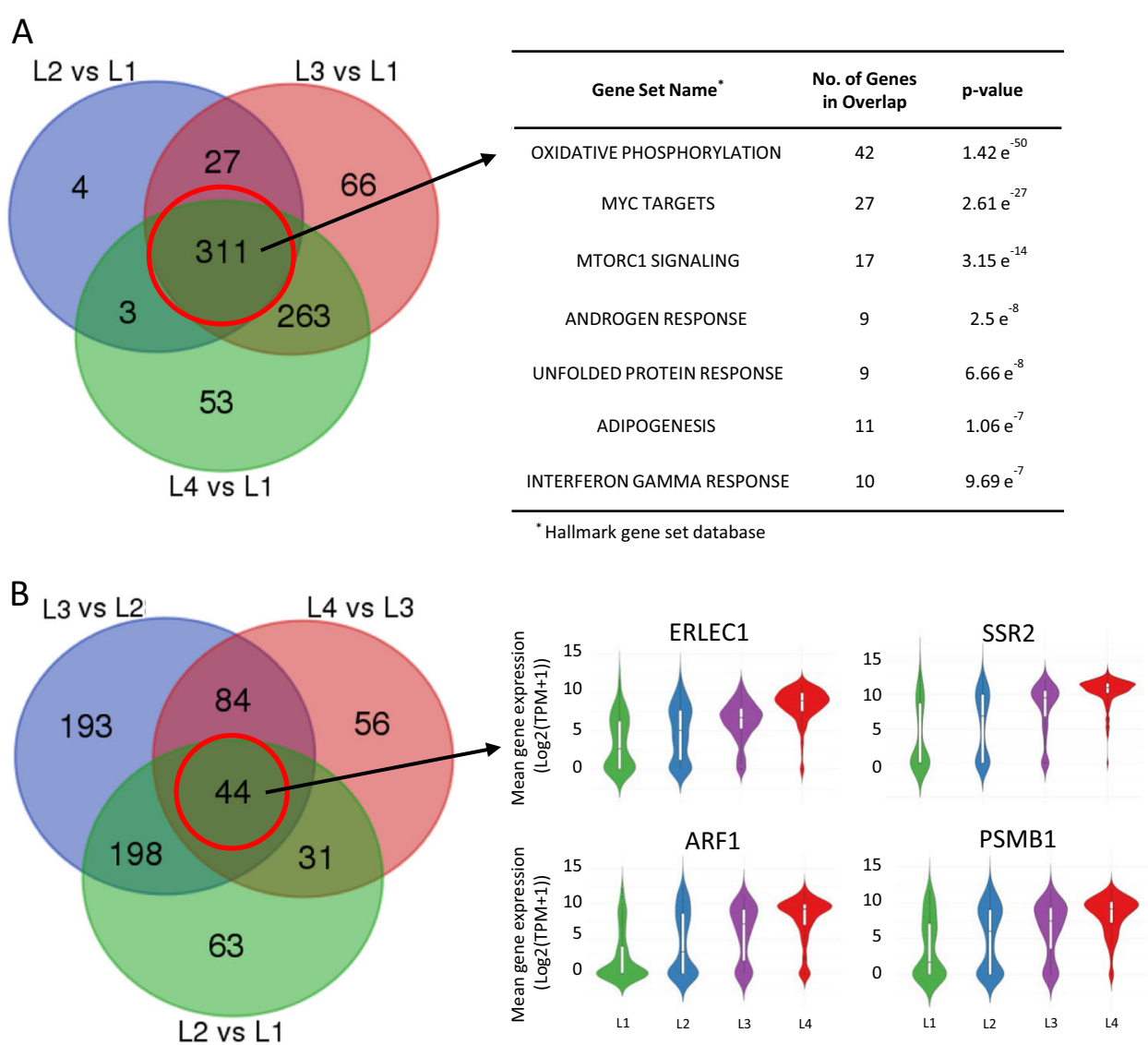

Fig. 4 Differential expression genes and associated pathways with MM Progression. a Most significantly up-regulated $(F C \geq 2, p<0.05)$ and shared 311 genes when comparing each cell groups to L1. $\mathbf{b}$ Identification of 44 genes with most consistently altered in expression levels (FC $\geq 2, p<$ 0.05 ) between the adjacent groups and sample violin plots for 4 of 44 shared genes (red circle)
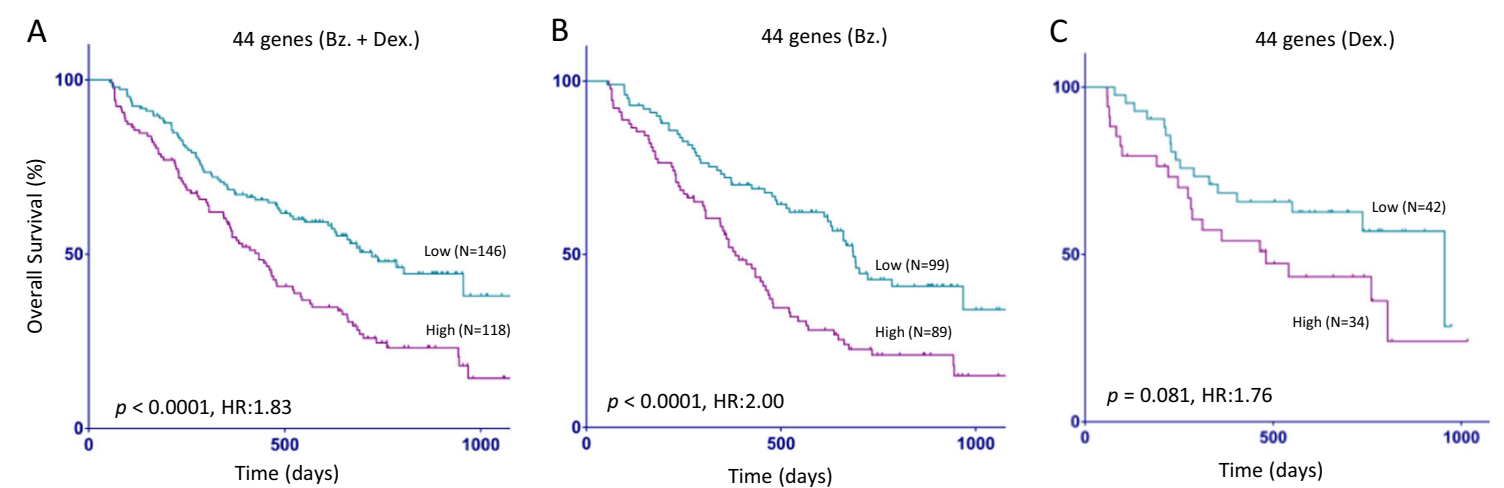

Fig. 5 Survival analysis using $\mathbf{4 4}$ signature gene sets. Microarray gene expression data from APEX (a-c) was used and Kaplan-Meier (KM) survival curve are shown based on the high and low expression status of the signature genes. $p$-values were generated using Mantel-Cox log-rank test. Bz. Bortezomib; Dex. Dexamethasone, HR hazard ratio, $Y$-axis percentage of survival, $X$-axis days of survival from randomization

levels. Although a relatively small study involving a few hundred cells, most cells in the L1 group displayed a relatively the lowest level of activations for genes involved in oxidative phosphorylation and proteasome homeostasis (Figs. 3a and 4a). L2 and L3 contained a majority of the cells from patients with trisomies and cytogenetically complexed MM (Table 1). L4 group is exclusive of cells from patients with $t(4 ; 14)$. It is worth noting that proteasome genes were some of the most prominently expressed in L3 and L4 groups and their expression was 
successively increased compared to cells in the L1 and L2 groups (Fig. 3). These results are consistent with the favorable responses observed in some MM patients treated with proteasome inhibitor.

Recently, there is increasing evidence of interplay between UPR and the mammalian target of rapamycin (mTOR) kinase signaling pathway ${ }^{24-26}$. Both mTOR and UPR pathways control many cellular processes including apoptosis, translation, energy metabolism, and inflammation $^{24-27}$. mTOR regulates cell growth, survival, proliferation, and metabolism. In this study, we observed that mTORC1 pathway genes were not only significantly enriched and elevated in the high-risk cell cluster, but also likely serve as a distinct feature with activation of UPR, glycolysis, and protein secretion pathways between the high risk of trisomies MM (L3) and $t(4 ; 14)$ RRMM (L4) groups (Table 1 and Supplemental Table S2). These observations are supported by several reports on the crosstalk between UPR and mTORC1 pathway in the cells contributing to malignancy cells ${ }^{14,16-18}$ and provide the mechanistic rationale to mTOR inhibitors as a useful regimen to potentially improve treatment efficacy for $\mathrm{MM}$ and RRMM in combination with proteasome inhibitors $^{24,26-28}$.

The clinical relevance of our single cell analysis is highlighted by the strong association of the 44 gene signature with OS from MM in independent clinical studies (Fig. 5). Significantly, 26 of the 44 genes consistently overexpressed among the four groups (Fig. 4b) were related to protein homeostasis and energy metabolism function and likely contributed to treatment response and $\mathrm{OS}$ in patients treated with proteasome inhibitors (Supplemental Table S5).

In conclusion, we used scRNA-Seq to examine gene expression signature of individual plasma cells $(n=597)$ from 15 patients at different stages of MM progression. We showed that different fractions of CD138-positive cells from each of the 15 patients clustered into four main groups corresponding to increasing risk levels in MM. Compared to the minimal risk cluster which contained most of the plasma cells derived from MGUS patients, increased expression of genes involved in protein homeostasis in MM cells in the later groups (L2-L4) is associated with progression and reduced survival of MM. Although our study is limited to only 15 patients and a total of 597 cells, comprehensive bioinformatics analyses of this high-resolution molecular dataset enabled us to derive at a robust molecular signature in MM progression at the resolution of single cells that reflect different risk levels across all samples. Our findings will require validation in a larger patient cohort, use of sequentially obtained plasma cells during the course of MGUS to MM progression, as well as improvements in analytical capabilities by analyzing many more cells per patients and the inclusion of unique molecular indexes (UMIs) during the cDNA synthesis to validate whether combination regimens including proteasome inhibitors and mTOR inhibitors could improve MM treat and overcome drug resistance in MM and RRMM. It will also be highly informative to determine whether the fraction of plasma cells within different risk clusters change over time in individual MM patients and how they affect disease progression, treatment response, and patient outcome.

\begin{abstract}
Acknowledgements
This work was supported in part by the Mayo Clinic_-University of Minnesota Partnership Award, Mayo Clinic Cancer Center, and Center for Individualized Medicine A Abyzov and J Jen. We thank members of the Genome Analysis Core for technical support over the course of this study. Special thanks to Dr. Liguo Wang for valuable discussions on TIN calculation and Aditya Bhagwate for initial data processing. Helpful discussions with Drs. HP Li and S Prabhakar regarding the Reference component analysis approach are also appreciated. Current address for Lintao Bi is Department of Hematology and Oncology, China-Japan Union Hospital, JiLin University, JiLin, China. Current address for Jin Jen is Celgene Corporation, 10300 Campus Point, San Diego, CA92121.
\end{abstract}

\section{Author details}

'Genome Analysis Core, Medical Genome Facility, Center for Individualized Medicine, Mayo Clinic, Rochester, MN, USA. ${ }^{2}$ Division of Bioinformatics and Biostatistics, Department of Health Science Research, Mayo Clinic, Rochester, MN, USA. ${ }^{3}$ Department of Drug Discovery and Development, Harrison School of Pharmacy, Auburn University, Auburn, AL, USA. ${ }^{4}$ Division of Hematology, Department of Internal Medicine, Mayo Clinic, Rochester, MN, USA.

${ }^{5}$ Department of Orthopedic Surgery, Mayo Clinic, Rochester, MN, USA.

${ }^{6}$ Department of Laboratory Medicine and Pathology, Mayo Clinic, Rochester, MN, USA. 'Department of Genetics, Cell Biology \& Development, University of Minnesota, Minneapolis, MN, USA

\section{Author contributions}

J.J., S.K. and B.V.N. designed the study. J.S.J. performed experimental research, analyzed the data, and drafted manuscript; Y.L. and A.A. performed bioinformatics analysis; S.K., S.V.R., L.B. and L.B.B. provided all samples and clinical data used in the study; A.M. performed survival analysis; and J.S.J., Y.L., J.J. and A.J.v.W. revised the manuscript with contribution from all co-authors.

\section{Conflict of interest}

The authors declare that they have no conflict of interest.

\section{Publisher's note}

Springer Nature remains neutral with regard to jurisdictional claims in published maps and institutional affiliations.

Supplementary Information accompanies this paper at (https://doi.org/ 10.1038/s41408-018-0160-x).

Received: 11 June 2018 Revised: 17 August 2018 Accepted: 31 August 2018 Published online: 03 January 2019

\section{References}

1. Rajkumar, S. V. \& Kumar, S. Multiple myeloma: diagnosis and treatment. Mayo Clin. Proc. 91, 101-119 (2016).

2. Morgan, G. J., Walker, B. A. \& Davies, F. E. The genetic architecture of multiple myeloma. Nat. Rev. Cancer 12, 335-348 (2012).

3. Broyl, A. et al. Gene expression profiling for molecular classification of multiple myeloma in newly diagnosed patients. Blood 116, 2543-2553 (2010). 
4. Zingone, A. \& Kuehl, W. M. Pathogenesis of monoclonal gammopathy of undetermined significance and progression to multiple myeloma. Semin. Hematol. 48, 4-12 (2011).

5. Bolli, N. et al. Heterogeneity of genomic evolution and mutational profiles in multiple myeloma. Nat. Commun. 5, 2997 (2014).

6. Melchor, L. et al. Single-cell genetic analysis reveals the composition of initiating clones and phylogenetic patterns of branching and parallel evolution in myeloma. Leukemia 28, 1705-1715 (2014).

7. Mitra, A. K. et al. Single-cell analysis of targeted transcriptome predicts drug sensitivity of single cells within human myeloma tumors. Leukemia $\mathbf{3 0}$ 1094-1102 (2016).

8. Kalari, K. R. et al. MAP-RSeq: mayo analysis pipeline for RNA sequencing. BMC Bioinforma. 15, 224 (2014).

9. Wang, L. et al. Measure transcript integrity using RNA-seq data. BMC Bioinform. 17, 58 (2016).

10. Liu, Q. et al. RNA-seq data analysis at the gene and CDS levels provides a comprehensive view of transcriptome responses induced by 4 hydroxynonenal. Mol. Biosyst. 9, 3036-3046 (2013).

11. Satija, R., Farrell, J. A., Gennert, D., Schier, A. F. \& Regev, A. Spatial reconstruction of single-cell gene expression data. Nat. Biotechnol. 33, 495-502 (2015).

12. Shalek, A. K. et al. Single-cell transcriptomics reveals bimodality in expression and splicing in immune cells. Nature 498, 236-240 (2013).

13. Hsiao, L. L. et al. A compendium of gene expression in normal human tissues. Physiol. Genom. 7, 97-104 (2001).

14. Richardson, P. G. et al. Extended follow-up of a phase 3 trial in relapsed multiple myeloma: final time-to-event results of the APEX trial. Blood 110, 3557-3560 (2007).

15. Barlogie, B. et al. Curing myeloma at last: defining criteria and providing the evidence. Blood 124, 3043-3051 (2014).

16. Hartigan, J. A. \& Wong, M. A. Algorithm AS 136: a K-means clustering algorithm. J. R. Stat. Soc. 28, 100-108 (1979).
17. $\mathrm{Li}, \mathrm{H}$. et al. Reference component analysis of single-cell transcriptomes elucidates cellular heterogeneity in human colorectal tumors. Nat. Genet. 49, 708-718 (2017)

18. Dalerba, P. et al. Single-cell dissection of transcriptional heterogeneity in human colon tumors. Nat. Biotechnol. 29, 1120-1127 (2011).

19. Wu, A. R. et al. Quantitative assessment of single-cell RNA-sequencing methods. Nat. Methods 11, 41-46 (2014).

20. Kolodziejczyk, A. A., Kim, J. K., Svensson, V., Marioni, J. C. \& Teichmann, S. A. The technology and biology of single-cell RNA sequencing. Mol. Cell 58, 610-620 (2015).

21. Kim, K. T. et al. Single-cell mRNA sequencing identifies subclonal heterogeneity in anti-cancer drug responses of lung adenocarcinoma cells. Genome Biol. 16, 127 (2015).

22. Pollen, A. A. et al. Low-coverage single-cell mRNA sequencing reveals cellular heterogeneity and activated signaling pathways in developing cerebral cortex. Nat. Biotechnol. 32, 1053-1058 (2014).

23. Kalisky, T., Blainey, P. \& Quake, S. R. Genomic analysis at the single-cell level. Annu. Rev. Genet. 45, 431-445 (2011).

24. Polak, P. \& Hall, M. N. mTOR and the control of whole body metabolism. Curr. Opin. Cell Biol. 21, 209-218 (2009).

25. Hotamisligil, G. S. Endoplasmic reticulum stress and the inflammatory basis of metabolic disease. Cell 140, 900-917 (2010).

26. Appenzeller-Herzog, C. \& Hall, M. N. Bidirectional crosstalk between endoplasmic reticulum stress and mTOR signaling. Trends Cell Biol. 22, 274-282 (2012).

27. Dazert, E. \& Hall, M. N. mTOR signaling in disease. Curr. Opin. Cell Biol. 23, 744-755 (2011).

28. Chen, $\mathrm{X}$. et al. Genetic and pharmacologic evidence that mTOR targeting outweighs mTORC1 inhibition as an antimyeloma strategy. Mol. Cancer Ther. 13, 504-516 (2014). 